\title{
Plasma-Etching of the Organic Layer in Nacre
}

\author{
Kirsten Parratt, Johnathan M. Yao, Gerald R. Poirier, Nan Yao* \\ Princeton Institute for the Science and Technology of Materials, Princeton University, New Jersey, USA \\ Email: ${ }^{*}$ nyao@princeton.edu
}

Received 22 April 2014; revised 22 May 2014; accepted 24 June 2014

Copyright (C) 2014 by authors and Scientific Research Publishing Inc.

This work is licensed under the Creative Commons Attribution International License (CC BY). http://creativecommons.org/licenses/by/4.0/

(c) (i) Open Access

\begin{abstract}
Nacre's brick and mortar structure has been motivating innovations in biomimetic materials for decades. However, there is still room to improve understanding of the structure of the organic layer in order to engineer better biomimetic composites. A plasma-etching technique that allows for the selective removal of some organic components from individual layers is developed. We conclude that this technique enables a closer examination of the organic layer such that the locations and mechanical properties of individual components can be determined. A methodology for examining nacre samples that have not been demineralized provides a more accurate substrate for understanding the structure-property relationships of the organic layer in native nacre.
\end{abstract}

\section{Keywords}

Plasma-Etching, Nacre, Haliotis rufescens, $\beta$-Chitin, AFM, SEM

\section{Introduction}

For many years nacre has been a source of biomimetic inspiration for the materials community due to its hierarchical structure and organic template-assisted self-assembly [1]. Nacre can be best visualized as a "brick and mortar" structure in which the bricks are composed of aragonite and the mortar consists of a variety of polysaccharides and protein fibers [2]. The exact structure of these two components, and the manner in which they interact to provide nacre with an array of interesting deformation mechanisms, has been the subject of scientific investigation for many years [3]-[9].

In order to simplify the discussion surrounding the interaction between the inorganic and organic layers, there has also been work focused on improving understanding of the structure of the organic layer. Song et al. have examined the structural and mechanical characteristics of organic matrix layers, and discussed how their find*Corresponding author. 
ings relate to the theory of the existence of mineral bridges between adjacent inorganic layers [10]. Sumitomo et al. used TEM to study the deformation behavior of the organic layer under strain and found that it demonstrates a crack-bridging toughening mechanism as well as some reversible deformation [10]. Bezares et al. demineralized nacre samples and suggested that the organic layer might consist of intercalcated silk fibroin/chitin planes that contribute to shear lag and protein connectivity [7]. There has even been biomineralization work that tests the ability of proteins and polysaccharides from nacre's organic layer to induce biomineral formation [11].

The microscopy community has also contributed work on structure-function relationships in the organic layer of nacre. Recent advances in imaging technology such as high-resolution transmission electron microscopy (HRTEM) and cryo-transmission electron microscopy (Cryo-TEM) have enabled scientists to more closely examine the organic layer than was previously possible. Yao et al. used HRTEM to show that many "mineral bridges" are in fact discontinuous but abutting nanoasperities [4]. Levi-Kalisman used cryo-TEM to investigate the interlamellar sheets and demonstrate that they consist of highly ordered and aligned $\beta$-chitin fibrils [12]. Meyers et al. took force measurements of both the dry and the hydrated organic layer to show that the process of drying lends the organic layer more strength and brittleness, suggesting a glass transition of the incorporated chitin [6]. Finally, AFM work by Liu et al. posited that the organic layer might in fact consist of multiple, mechanically-distinct organic sublayers [5]. However, nacre is structured such that these organic layers often do not cleave apart easily and therefore hinders the independent observation of different layers. The goal of this project was to investigate further the possibility of sublayers by developing a plasma-etching technique that could selectively remove organic layers.

Plasma-etching has been used before in the context of research on nacre, however, it has thus far served only to clean samples for the examination of the inorganic layer [12]-[17]. This project is therefore believed to be the first instance in which plasma-etching was used to investigate the components of the organic layer of gastropod nacre.

\section{Materials and Methods}

In these experiments, gastropod nacre was sampled from Haliotis rufescens. This type of nacre is organized in columnar form and demonstrates a lamellar structure [18]. Columnar indicates that each polygonal tablet is of similar shape and size, and that the centers of the stacked tablets roughly align [3]. Nacre is built up of alternating layers of inorganic material and organic material which can be seen in Figure 1.

The inorganic layers are made of calcium carbonate in the form of aragonite tablets which are $0.5 \mu \mathrm{m}$ thick and approximately $5 \mu \mathrm{m}$ in diameter [3]. These are indicated by the white areas of Figure 1. The organic layers are much thinner, only $20 \mathrm{~nm}$ under compression [18], and composed of a variety of proteins and polysaccharides [3] with a central core of $\beta$-chitin [1]. The organic material is indicated by the black lines in Figure 1. Samples were originally prepared via the methodology developed in Wang et al. [18]. However, one deviation from this methodology is that all samples used in this study were both stored and tested in air.

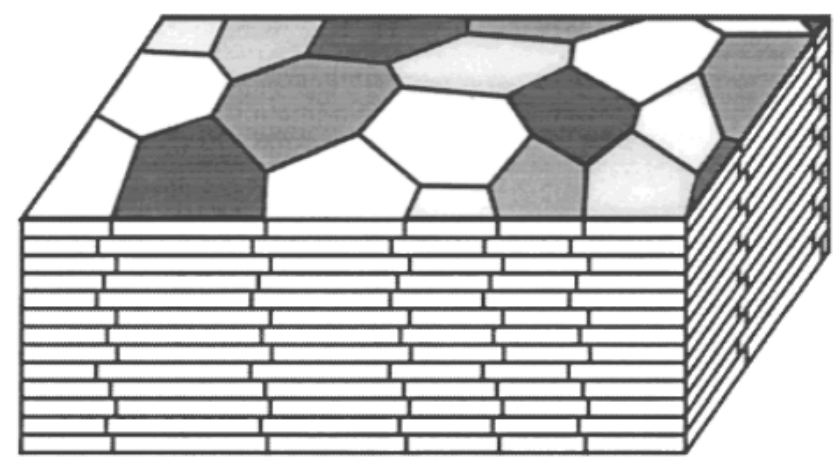

Figure 1. Illustration of the columnar structure of abalone shell. Columnar nacre is differentiated from sheet nacre by the tessellated arrangement of the intertablet boundaries that arises due to the alignment of the stacked tablet centers. (Reproduced from [16]). 
Samples that were approximately $2 \mathrm{~mm}$ by $1 \mathrm{~mm}$ across and several hundred microns thick were prepared to be plasma-etched and imaged. First, a handheld vacuum pickup tool was used to place each nacre sample on a mounting adhesive attached to an AFM sample disk. Scotch tape was then pressed on the nacre surface in order to cleave the top layers off the sample and expose a fresh surface. This was repeated many times, until the sample disk was covered, in order to uncover multiple sites of interest. AFM and SEM were then used to provide preliminary images of the untreated sample surface. The AFM used was a Nanoman (Digital Instruments) and the SEM used was a FEI Quanta 200 FEG Environmental-SEM. In order to capture SEM images of an uncoated nacre sample, low vacuum examination was performed at 0.75 Torr with a $15 \mathrm{kV}$ beam.

The system used was a radio frequency glow discharge plasma source which can generate a large volume of stable plasma and is illustrated in Figure 2.

Plasma-etching was performed in a PDC-32G Harrick Plasma Cleaner with a feed gas composed of argon (79\%) and oxygen (21\%). The samples were plasma-etched at low pressure in one minute increments and imaged between each etching to track changes in the organic layer. In order to reliably track changes, an area of interest was chosen in the preliminary SEM imaging session. Images were then taken of the area at successively lower magnification such that the area could be found quickly and easily after each plasma-etching.

\section{Results and Discussion}

In order to remove organic material from the organic layers of nacre, the technique developed here relies on the degradation reaction that results from plasma-etching. Oxygen plasma-etching rates are linearly proportional to the oxygen atomic concentration in the plasma and the process is initiated by oxygen free radicals [19]. Weight loss from degradation is dependent on the energy of the plasma and the number of oxygen functionalities and strong backbone bonds present in the polymer [19] [20]. Additionally, weight loss will be restricted to the topmost layers of the polymer [20]. This can be seen in Figure 3 which shows the progression of the degradation of the organic layer covering a single nacre platelet with incremental plasma-etching.

Figure 3 shows that over time the plasma-etching cause small holes to develop in the organic layer of the nacre. Initially in Figure 3(a) the organic layer was smooth with no indications of where the holes will develop. In Figure 3(b) small, pale circles have begun to emerge that resemble globules. However, continued plasma-etching yields Figure 3(c) and Figure 3(d) which clearly show the holes that have been opened in the organic matrix and Figure 3(e) and Figure 3(f) show that the plasma-etching continues deeper into the organic layer to reveal more holes in the organic matrix. These holes have diameters between $10-50 \mathrm{~nm}$ and are believed to be pores in the chitin mesh.

Previous work by other groups has proven the existence of "holes" or "pores" in the organic layer. By demineralizing nacre samples, it has been shown that the inorganic component of nacre can be removed while leaving several components of the organic scaffolding intact [5] [6] [19]. Meyers et al. concluded that the holes result from a fibril matrix of chitin macromolecules, which form the structural component of the intertile organic layer [6]. Blank et al. took AFM images of demineralized multilamellar organic sheets concluded that the fibrous network of chitin contains pores of $40-80 \mathrm{~nm}$ in diameter [21]. They then incubated the demineralized organic sheets with proteinase $\mathrm{K}$ for two hours and found that the pore size increased slightly. However, cryoTEM work by Levi-Kalisman et al. pores were found to have a diameter of approximately $5 \mathrm{~nm}$ in diameter and

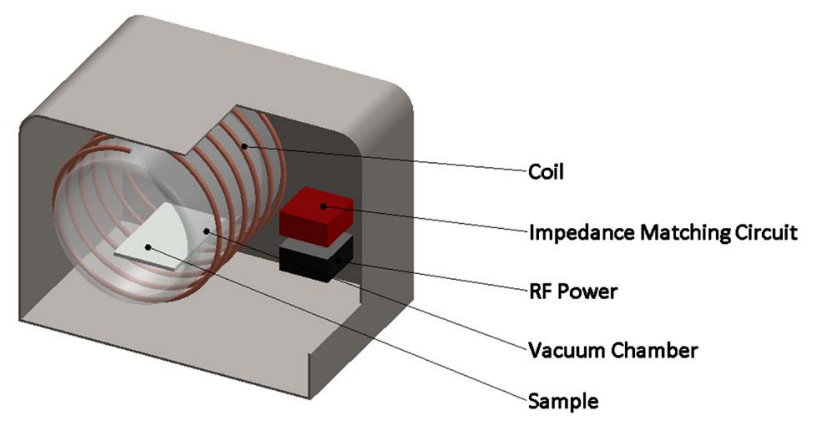

Figure 2. Schematic of the radio frequency glow discharge plasma-etching system. 

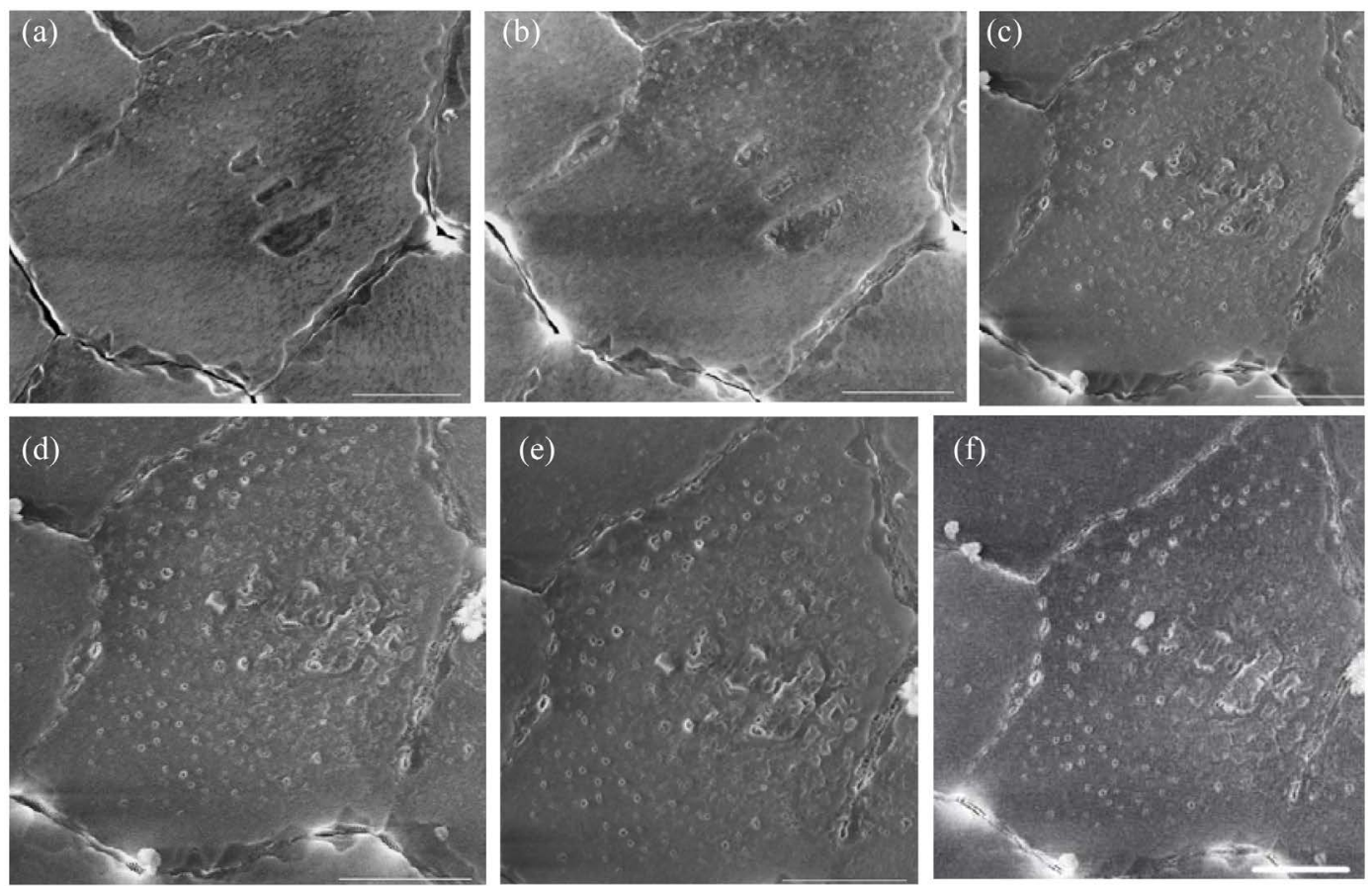

Figure 3. Samples that have been plasma-etched for (a) 1 minute; (b) 2 minutes; (c) 3 minutes; (d) 4 minutes; (e) 5 minutes; (f) 7.5 minutes. All scale bars represent $1 \mu$ m length.

they suggest that microscopy techniques necessarily involve dehydration and therefore the introduction of artifacts into the data [12].

The distance between pores was determined by Schaffer et al. who grew flat pearls and then fixed demineralized interlamellar organic sheets [22]. They measured a distance of $20-100 \mathrm{~nm}$ between these holes in the organic layer. Normally the chitin fibrils are contained between layers of acidic macromolecules but these have been etched away during the demineralization process. Figure 3 shows that, like demineralization, plasmaetching is capable of revealing the chitin core of the organic layers of nacre. However, unlike demineralization, it is possible that plasma-etching can be more precise and remove only the organic components that are most susceptible to degradation.

Figure 4 offers a higher magnification of Figure 3(d) which better demonstrates the distribution of the pores in the organic layer across a single platelet and the nature of individual pores. The image shows the nacre sample after it has undergone four minutes of plasma-etching and the holes in the uppermost part of the chitin layer are clearly visible. Bezares et al. proposed that the organic layer consists of an intercalcated chitin/silk fibroin core sandwiched between layers of acidic macromolecules [7]. Our work suggests that the etching process gradually removes the acidic macromolecules from the chitin mesh as the macromolecules react with the plasma, and therefore leaves behind the characteristic pore structure. Chitin and silk fibroin both have a low susceptibility to plasma-etching compared to the acidic macromolecules. This is because the acidic proteins in the organic layer are thought to have anionic side chains similar to the spacing of calcium in calcium carbonate crystals thereby giving them a role in aragonite nucleation [21].

\section{Conclusion}

In this study, to our knowledge for the first time, plasma-etching has been used to investigate the organic layer of gastropod nacre rather than to remove it entirely to allow for examination of the inorganic layer. By plasma-etching the organic layer of nacre samples for one minute increments, we showed that the gradual decomposition of the organic layer could be observed with time. Different components of the organic layer were shown to etch at different rates due to the nature of oxygen plasma-etching, which allowed us to conclude that the chitin fibril mesh remained after being stripped of smaller, oxygen functionality-containing molecules such as perlustrin or perlucin. Based on this work, techniques could be developed to selectively remove the various com- 


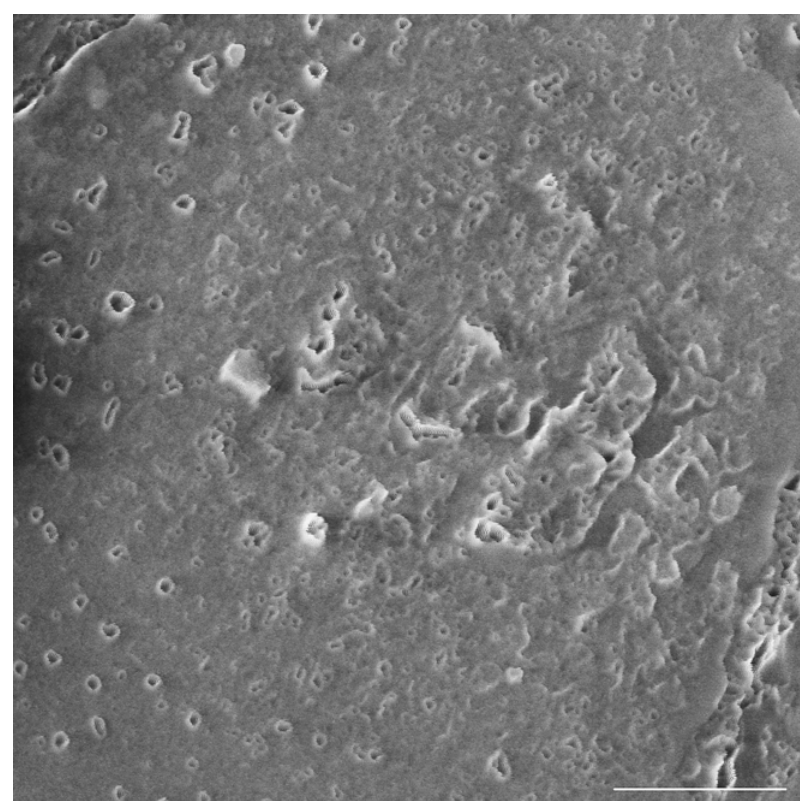

Figure 4. A higher magnification of Figure 3(d) which clearly shows the distribution of pores in the organic layer across the platelet's surface. The scale bar represents $500 \mathrm{~nm}$.

ponents of the organic layer so that the organic components can be examined individually rather than the organic layer as a whole. This would lead to easier mapping of the locations of different components and also to more precise measurement of the mechanical properties.

\section{Acknowledgements}

The authors acknowledge funding from Princeton University's Lidow research fund and the partial support of the National Science Foundation-MRSEC program through the Princeton Center for Complex Materials (DMR-0819860).

\section{References}

[1] Malik, A.S., Boyko, O., Atkar, N. and Young, W.F. (2001) A Comparative Study of MR Imaging Profile of Titanium Pedicle Screws. Acta Radiologica, 42, 291-293. http://dx.doi.org/10.1080/028418501127346846

[2] Aksay, I., Trau, M., Manne, S., Honma, I., Yao, N., Zhou, L., Fenter, P., Eisenberger, P. and Gruner, S. (1996) Biomimetic Pathways for Assembling Inorganic Thin Films. Science, 273, 892-898. http://dx.doi.org/10.1126/science.273.5277.892

[3] Addadi, L. and Weiner, S. (1997) A Pavement of Pearl. Nature, 389, 912-915. http://dx.doi.org/10.1038/40010

[4] Yao, N., Epstein, A., Liu, W., Sauer, F. and Yang, N. (2009) Organic-Inorganic Interfaces and Spiral Growth in Nacre. Journals of the Royal Society Interface, 6, 367-376. http://dx.doi.org/10.1098/rsif.2008.0316

[5] Liu, W. and Yao, N. (2008) Characterization of the Organic-Inorganic Interface of Abalone Shell Nacre. Microscopy and Microanalysis Proceedings, 14, 24-25. http://dx.doi.org/10.1017/S143192760808118X

[6] Meyers, M., Lim, C., Li, A., Nizam, B.H., Tan, E., Seki, Y. and McKittrick, J. (2009) The Role of Organic Intertile Layer in Abalone Nacre. Materials Science and Engineering C, 29, 2398-2410. http://dx.doi.org/10.1016/j.msec.2009.07.005

[7] Bezares, J., Asaro, R. and Hawley, M. (2010) Macromolecular Structure of the Organic Framework of Nacre in Haliotis rufescens: Implications for Mechanical Response. Journal of Structural Biology, 170, 484-500. http://dx.doi.org/10.1016/j.jsb.2010.01.006

[8] Katti, K. and Katti, D. (2006) Why is Nacre So Tough and Strong? Materials Science and Engineering C, 26, 13171324. http://dx.doi.org/10.1016/j.msec.2005.08.013

[9] Wise, S. and deVilliers, J. (1971) Scanning Electron Microscopy of Molluscan Shell Ultrastructures: Screw Disloca- 
tions in Pelecypod Nacre. Transactions of the American Microscopical Society, 90, 376-380. http://dx.doi.org/10.2307/3225200

[10] Sumitomo, T., Kakisawa, H., Owaki, Y. and Kagawa, Y. (2008) In Situ Transmission Electron Microscopy Observation of Reversible Deformation in Nacre Organic Matrix. Journal of Material Research, 23, 1466-1471. http://dx.doi.org/10.1557/JMR.2008.0184

[11] Song, F., Soh, A. and Bai, Y. (2003) Structural and Mechanical Properties of the Organic Matrix Layers of Nacre. Biomaterials, 25, 3623-3631. http://dx.doi.org/10.1016/S0142-9612(03)00215-1

[12] Levi-Kalisman, Y., Falini, G., Addadi, L. and Weiner, S. (2001) Structure of the Nacreous Organic Matrix of a Bivalve Mollusk Shell Examined in the Hydrated State Using Cryo-TEM. Journal of Structural Biology, 135, 8-17. http://dx.doi.org/10.1006/jsbi.2001.4372

[13] Metzler, R., Evans, J., Killian, C., Zhou, D., Churchill, T., Appathurai, N., Coppersnith, S. and Gilbert, P. (2010) Nacre Protein Fragment Templates Lamellar Aragonite Growth. Journal of the American Chemical Society, 132, 6329-6334. http://dx.doi.org/10.1021/ja909735y

[14] Liu, G., Zhao, D., Tomsia, A., Minor, A., Song, X. and Saiz, E. (2009) Three-Dimensional Biomimetic Mineralization of Dense Hydrogel Templates. Journal of the American Chemical Society, 131, 9937-9939. http://dx.doi.org/10.1021/ja903817z

[15] Barthelat, F., Li, C., Comi, C. and Espinosa, H. (2006) Mechanical Properties of Nacre Constituents and their Impact on Mechanical Performance. Journal of Materials Research, 21, 1977-1986. http://dx.doi.org/10.1557/jmr.2006.0239

[16] Dastjerdi, A.K., et al. (2012) The Weak Interfaces Within Tough Natural Composites: Experiments on Three Types of Nacre. Journal of the Mechanical Behavior of Biomedical Materials, 19, 50-60. http://dx.doi.org/10.1016/j.jmbbm.2012.09.004

[17] Kingsley, R., Gilder, R., LeGeros, R. and Watabe, N. (2003) Multimineral Calcareous Deposits in the Marine Alga Acetabularia Acetabulum (Chlorophyta; Dasycladaceae). Journal of Phycology, 39, 937-947. http://dx.doi.org/10.1046/j.1529-8817.2003.02169.x

[18] Wang, R., Evans, A., Suo, Z., Yao, N. and Aksay, I. (2001) Deformation Mechanisms in Nacre. Journal of Material Research, 16, 2485-2493. http://dx.doi.org/10.1557/JMR.2001.0340

[19] Egitto, F. (1990) Plasma Etching and Modification of Organic Polymers. Pure and Applied Chemistry, 62, $1699-1708$. http://dx.doi.org/10.1351/pac199062091699

[20] Chu, P., Chen, J., Wang, L. and Huang, N. (2002) Plasma-Surface Modification of Biomaterials. Materials Science and Engineering R, 36, 143-206. http://dx.doi.org/10.1557/JMR.2001.0340

[21] Blank, S., Arnoldi, M., Khoshnavaz, S., Treccani, L., Kuntz, M., Mann, K. and Grathwohl, G. (2003) The Nacre Protein Perlucin Nuceates Growth of Calcium Carbonate Crystals. Journal of Microscopy, 212, 280-291. http://dx.doi.org/10.1111/j.1365-2818.2003.01263.x

[22] Schaffer, T., Ionescu-Zaneeti, C., Proksch, R., Fritz, M., Walters, D., Almqvist, N., Zaremba, C., Belcher, A., Smith, B., Stucky, G., Morse, D. and Hansma, P. (1997) Does Abalone Nacre Form by Heteroepitaxial Nucleation or by Growth Through Mineral Bridges? Chemistry of Materials, 9, 1731-1740. http://dx.doi.org/10.1021/cm960429i 
Scientific Research Publishing (SCIRP) is one of the largest Open Access journal publishers. It is currently publishing more than 200 open access, online, peer-reviewed journals covering a wide range of academic disciplines. SCIRP serves the worldwide academic communities and contributes to the progress and application of science with its publication.

Other selected journals from SCIRP are listed as below. Submit your manuscript to us via either submit@scirp.org or Online Submission Portal.
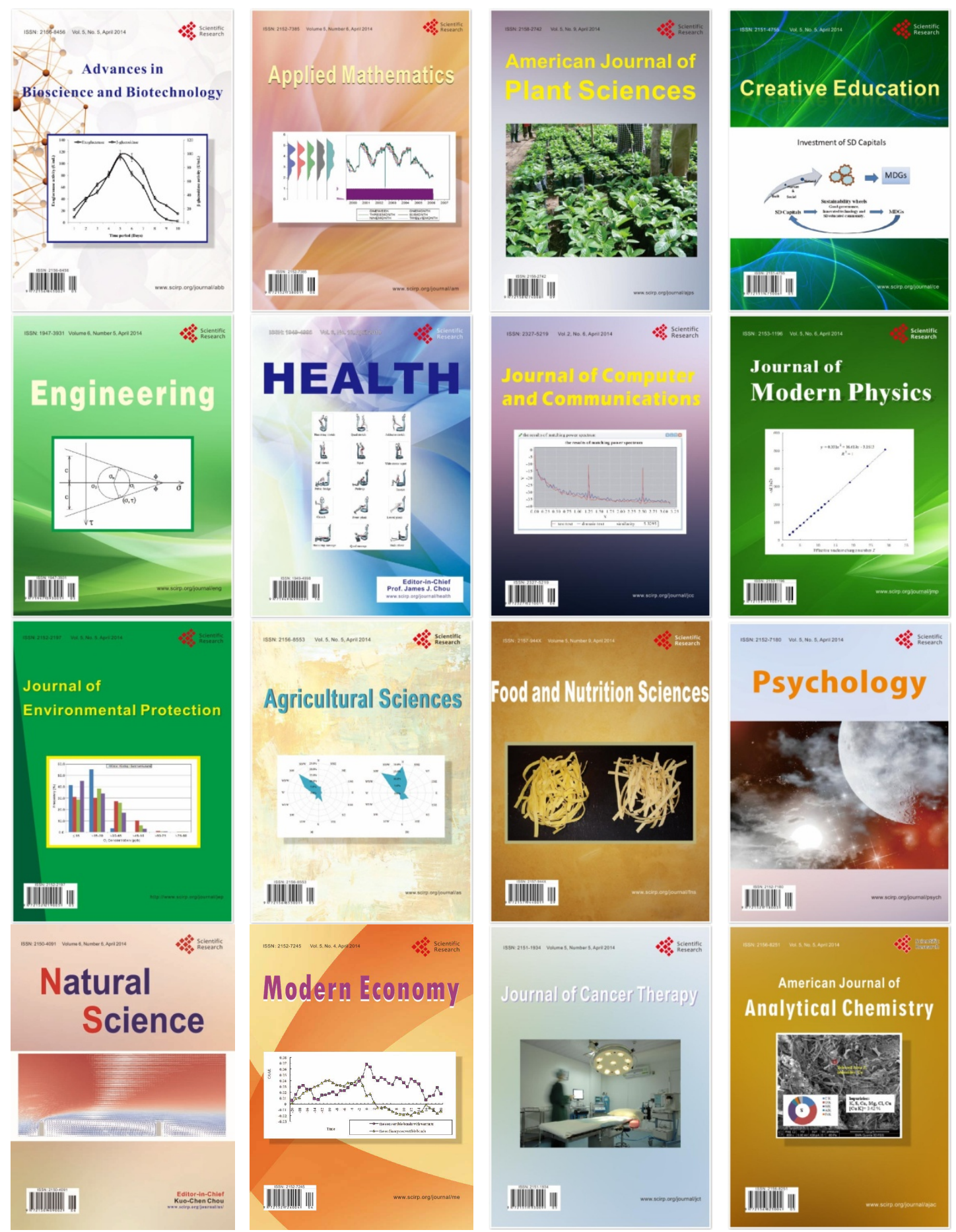